\title{
Rupture of the Free Left Ventricular Wall: A Novel Approach for Reconstruction
}

\author{
Felix Fleißner $^{1} \quad$ Jan D. Schmitto $^{1} \quad$ L. Christian Napp ${ }^{2} \quad$ Issam Ismail ${ }^{1}$ \\ ${ }^{1}$ Department of Cardiac, Thoracic, Transplantation and Vascular \\ Surgery, Hannover Medical School, Hannover, Germany \\ 2 Department of Angiology and Cardiology, Hannover Medical School, \\ Hannover, Germany \\ Address for correspondence Felix Fleißner, MD, Department of \\ Cardiac, Thoracic, Transplantation and Vascular Surgery, Hannover \\ Medical School, Carl-Neuberg- Strasse 1, Hannover 30625, Germany \\ (e-mail: fleissner.felix@mh-hannover.de).
}

Thorac Cardiovasc Surg Rep 2018;7:e30-e32.

\begin{abstract}
Keywords

- cardiopulmonary bypass

- coronary intervention

- extracorporeal membrane oxygenation

Background A rupture of the free wall of the left ventricle is a rarely seen complication of myocardial infarction and represents an absolute cardiac emergency.

Case Description We hereby present a case of a 64-year-old patient with a rupture of the free left ventricular wall. The patient was treated in an emergent operation with a novel reconstruction method of the left ventricular wall and was discharged 30 days after the initial operation.

Conclusion Left ventricular free wall rupture is rarely described in the literature, which might be because of high mortality in underdiagnosed cases. Therefore, early imaging by echo or computed tomography (CT) is essential for detecting this dangerous condition. Once diagnosed, urgent surgery is mandatory to save the life of the patient.
\end{abstract}

\section{Background}

With the widespread availability of catheterization laboratories and associated early coronary intervention in acute myocardial infarction, the incidence of left ventricular free wall rupture (LVFWR) has decreased over the past years. ${ }^{1-4}$ Nonetheless, reports conclude that still up to $2 \%$ of all cases with acute ST-elevation myocardial infarction present with this dreadful complication. ${ }^{5,6}$ Mortality remains very high, ${ }^{7}$ and survival of such an event depends on prompt cardiac surgery.

LVFWR is still associated with high mortality rates of up to $60 \%$ and is responsible for $95 \%$ of electromechanical dissociation in patients with acute myocardial infarction. ${ }^{8}$ Risk factors for the occurrence of left ventricular wall rupture occurs are age ( $>70$ years old), female sex, first myocardial infarction, ${ }^{2}$ initial high levels of myocardial biomarkers, ${ }^{3,6}$ and poor coronary collateralization. ${ }^{9}$

LVFWR is usually diagnosed by echocardiography, for which $100 \%$ sensitivity and $93 \%$ specificity have been reported, ${ }^{10}$ heart catheterization especially levocardiography, and computed tomography (CT).

received

February 2, 2018

accepted after revision

February 20, 2018

\section{Case Report}

A 64-year-old patient was admitted to our hospital after a car accident on the campus. He had been the driver and was on the way for an appointment in our outpatient clinic for trigeminal neuralgia. Prior to the accident, he had noticed severe dizziness. CT showed no signs of apoplexy or bleeding. Cardiac enzymes were elevated, and history taking revealed chest pain 3 days earlier. Therefore, he was scheduled for immediate cardiac catheterization. Coronary angiogram showed an occluded circumflex artery, and coronary intervention was not successful since the occlusion could not be passed with several wires. Ventriculography showed preserved systolic function of the left ventricle (LV) and a very weak and blurred contrast near to the lateral left ventricular wall (-Fig. 1), and hemoglobin levels had quickly dropped to $5.4 \mathrm{~g} / \mathrm{dL}$ according to blood gas analyses acquired during the catheterization procedure. Thus, urgent CT of thorax and abdomen was performed. CT showed no signs of pulmonary embolism and aortic dissection but confirmed rupture of the LV with a pseudoaneurysm of the LV and an associated large left-sided hematothorax (-Fig. 2).

\footnotetext{
(c) 2018 Georg Thieme Verlag KG Stuttgart · New York
}

License terms

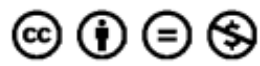




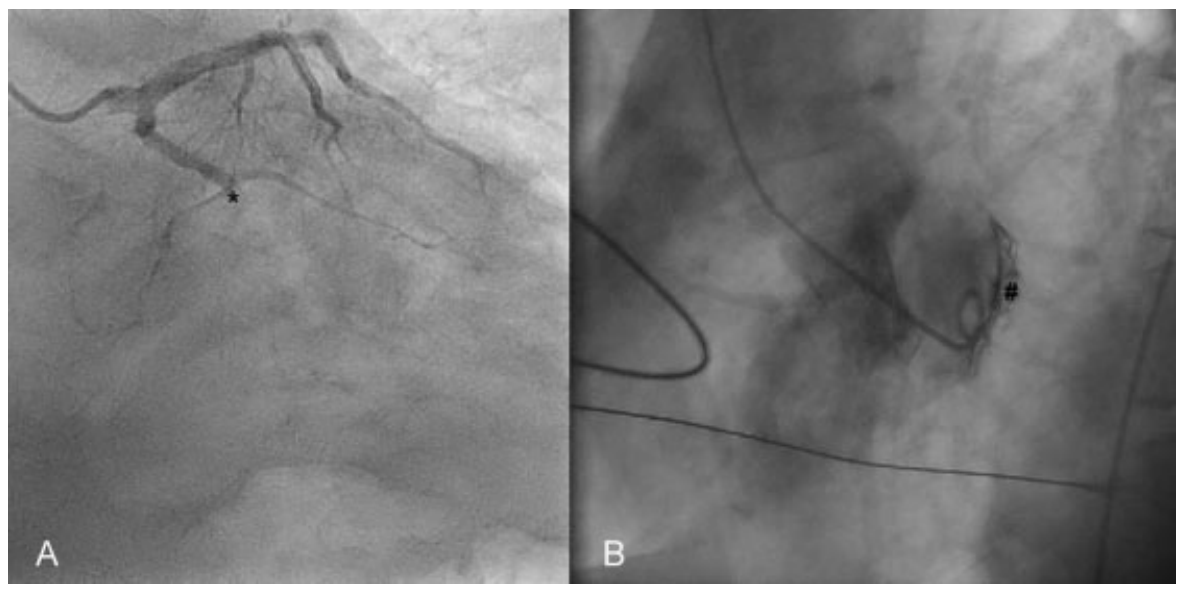

Fig. 1 (A) occluded circumflex artery (LAO 20/CAU 20) (*). (B) Levocardiography showing the pseudoaneurysm after the free left ventricular wall rupture (\#).

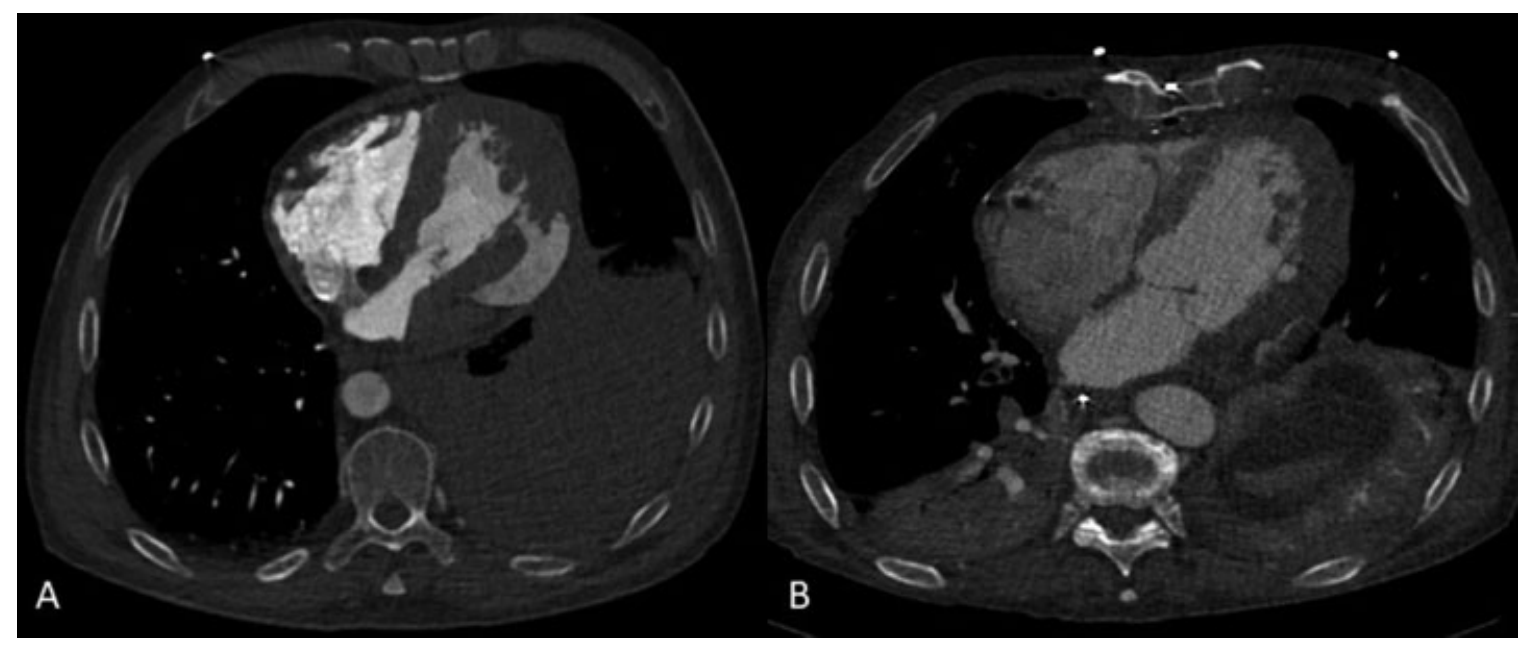

Fig. 2 (A) Computed tomography (CT) showing the pseudoaneurysm after left ventricular rupture and a large hematothorax. (B) CT after successful reconstruction of the left ventricle.

The patient was immediately transferred to the operating room and after median sternotomy was put on cardiopulmonary bypass. Pericardial adhesions were encountered and removed carefully. Then the aneurysm was resected. We found two entries leading to the large pseudoaneurysm. To spare the left ventricular wall and to avoid a large modified Dor plasty, we isolated both entries using two felt pledgets, each connected with running sutures ( $\mathbf{- F i g . ~ 3 ) . ~ A f t e r ~ s u c c e s s f u l ~ c l o s u r e ~ o f ~ b o t h ~ e n t r i e s , ~}$ we used a $4 \times 4 \mathrm{~cm}$ large bovine pericardial patch and BioGlue (Cryolife; without additional sutures) to prevent further bleeding (-Fig. 4).

To reduce left ventricular preload, the patient was placed on femorofemoral venoarterial extracorporeal membrane oxygenation (VA-ECMO) for the following 3 days. Afterward, VA-ECMO was weaned successfully, and the patient could be transferred to the normal ward on the eighth postoperative day. Postoperative transthoracic echocardiography showed a left ventricular ejection fraction of $25 \%$ with competent valves (-Fig. 4).

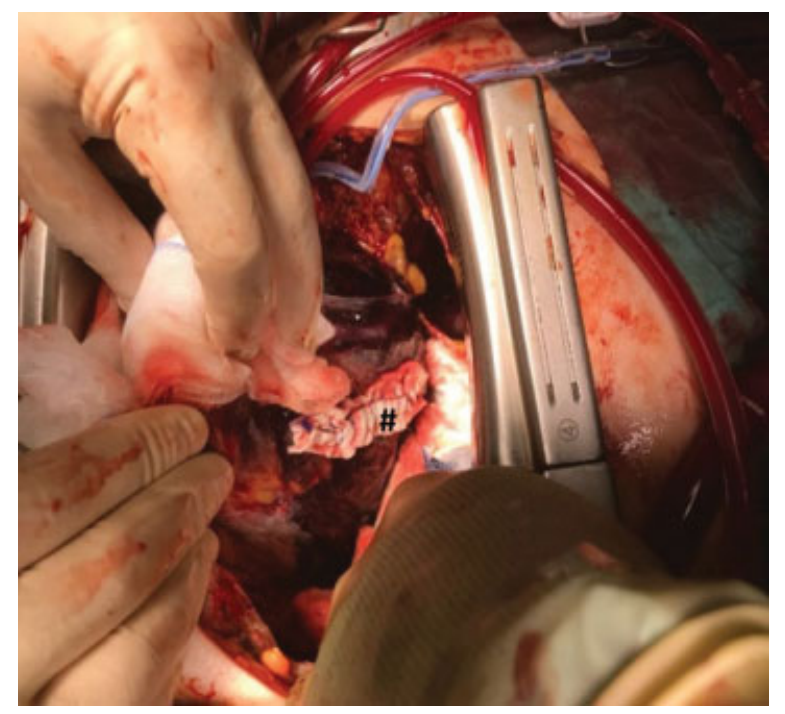

Fig. 3 The two felt pledgets covering the entry at the site just in line with the occluded circumflex artery. Later, additional two felt pledgets were sutured at the second entry site (\#). 


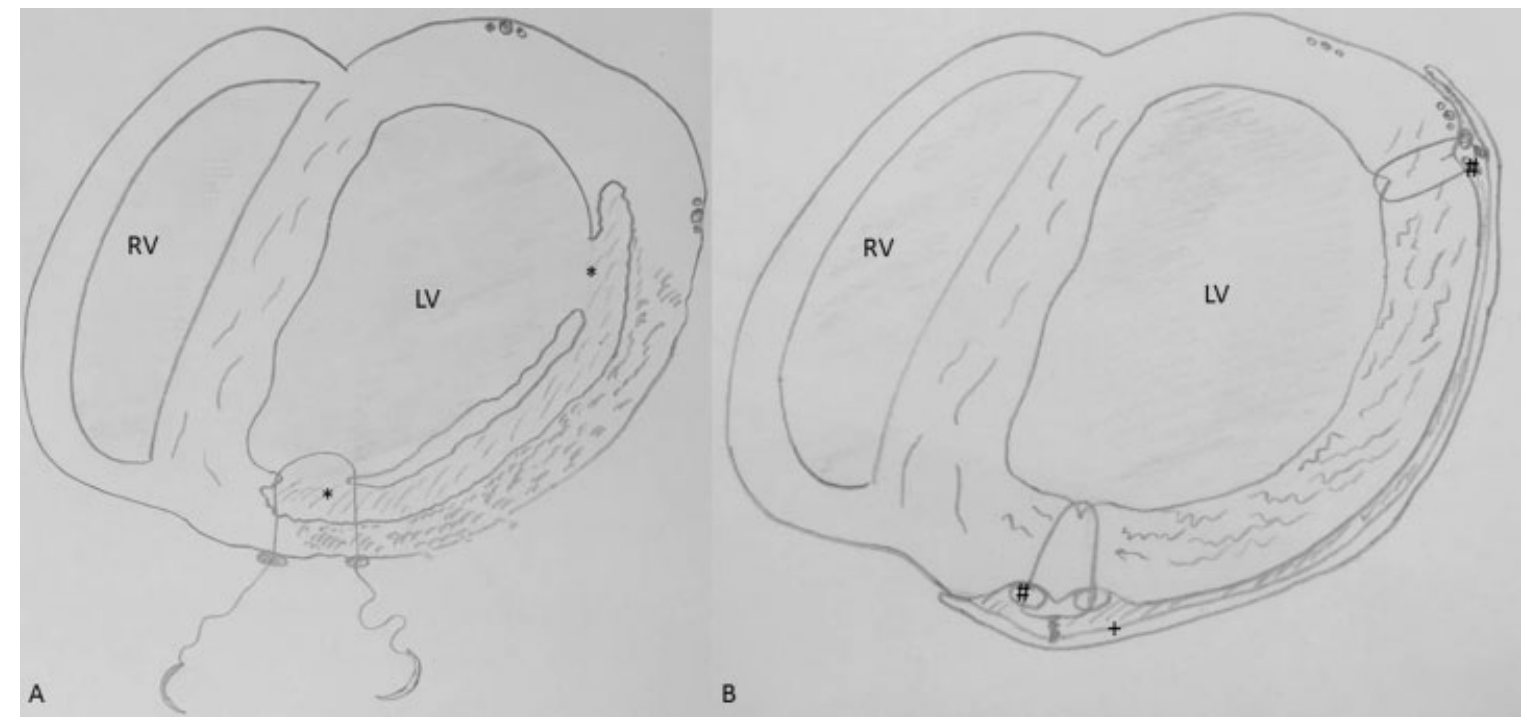

Fig. 4 (A) Scheme of used reconstruction method. Two large felt pledgets (\#) are sutured around the entry sites (*) of the pseudoaneurysm. (B) After the reconstruction, the entries are closed by the running suture. Additional BioGlue is used to attach a large pericardial patch $(+)$ on the outside to minimize bleeding. LV, left ventricle; RV, right ventricle.

Before discharge, the patient disclosed that he had had myocarditis during childhood, which explains the pericardial adhesions observed during surgery. Those adhesions most likely contributed to the favorable outcome by limiting blood loss through the large myocardial defects.

\section{Conclusion}

LVFWR has a high mortality and is very likely underdiagnosed in patients dying early after myocardial infarction. Our patient was lucky since he was already on the campus, was very early diagnosed, and had pericardial adhesions, which prevented complete rupture of the LV. Notwithstanding, we believe that our novel surgical method and postoperative mechanical circulatory support were essential for the favorable outcome of our patient. The usual problem with large Dor plasty is the fact that the acontractile plasty itself does not contribute to left ventricular function. Instead, our method with occlusion of the entries only aims to preserve as much myocardium as possible, which is the basis of anatomical reconstruction of the LV and long-term recovery.

\section{References}

1 Moreno R, López-Sendón J, García E, et al. Primary angioplasty reduces the risk of left ventricular free wall rupture compared with thrombolysis in patients with acute myocardial infarction. J Am Coll Cardiol 2002;39(04):598-603
2 Honda S, Asaumi Y, Yamane T, et al. Trends in the clinical and pathological characteristics of cardiac rupture in patients with acute myocardial infarction over 35 years. J Am Heart Assoc 2014; 3(05):e000984

3 Hosseinzadeh-Maleki M, Valizadeh N, Rafatpanah N, Moezi SA. Survival after left ventricular free wall rupture due to acute myocardial infarction. ARYA Atheroscler 2015;11(05):310-313

4 Fent G, Grech E, Parviz Y, Briffa N. Left ventricular free wall rupture complicating acute STEMI. Acute Card Care 2015;17(03): 45

5 Che J, Li G, Chen K, Liu T. Post-MI free wall rupture syndrome. Case report, literature review, and new terminology. Clin Case Rep 2016;4(06):576-583

6 Garg P, Abdel-Rahman SE, Greenwood JP, Plein S. Free-wall rupture post-reperfused acute myocardial infarction: insights from multimodality cardiovascular imaging. Circulation 2015; 132(21):e245-e247

7 French JK, Hellkamp AS, Armstrong PW, et al. Mechanical complications after percutaneous coronary intervention in ST-elevation myocardial infarction (from APEX-AMI). Am J Cardiol 2010; 105(01):59-63

8 Figueras J, Curós A, Cortadellas J, Soler-Soler J. Reliability of electromechanical dissociation in the diagnosis of left ventricular free wall rupture in acute myocardial infarction. Am Heart J 1996; 131(05):861-864

9 Yip HK, Wu CJ, Chang HW, et al. Cardiac rupture complicating acute myocardial infarction in the direct percutaneous coronary intervention reperfusion era. Chest 2003;124(02):565-571

10 López-Sendón J, González A, López de Sá E, et al. Diagnosis of subacute ventricular wall rupture after acute myocardial infarction: sensitivity and specificity of clinical, hemodynamic and echocardiographic criteria. J Am Coll Cardiol 1992;19(06):1145-1153 